\title{
Preventing heart injury during negative pressure wound therapy in cardiac surgery: Assessment using real-time magnetic resonance imaging
}

\author{
Malin Malmsjö, MD, PhD, ${ }^{a}$ Rainer Petzina, MD, ${ }^{\text {add }}$ Martin Ugander, MD, PhD, ${ }^{\mathrm{b}}$ Henrik Engblom, MD, \\ $\mathrm{PhD},{ }^{\mathrm{b}}$ Christian Torbrand, MD, ${ }^{\mathrm{a}}$ Arash Mokhtari, MD, PhD, ${ }^{\mathrm{c}}$ Roland Hetzer, MD, PhD,${ }^{\mathrm{d}}$ \\ Håkan Arheden, $\mathrm{MD}, \mathrm{PhD},{ }^{\mathrm{b}}$ and Richard Ingemansson, $\mathrm{MD}, \mathrm{PhD}^{\mathrm{c}}$
}

Objective: Heart rupture is a devastating complication to negative pressure wound therapy in cardiac surgery. Also, reduced cardiac output during negative pressure wound therapy has been reported. The present study aimed to examine the effects of negative pressure wound therapy on the position of the heart in relation to the thoracic wall using magnetic resonance imaging in a porcine sternotomy wound model.

\begin{abstract}
Methods: Six pigs had median sternotomy followed by negative pressure wound therapy at $-75,-125$, and -175 $\mathrm{mm} \mathrm{Hg}$. Real-time magnetic resonance imaging movies (10 images/s) were acquired in a midventricular transverse plane or a midsagittal plane during the application of negative pressure wound therapy.

Results: Similar finding were observed at all different negative pressures studied. Negative pressure wound therapy caused the heart to be displaced toward the thoracic wall, and in some cases, the right ventricular free wall bulged into the space between the sternal edges, and the sharp edges of the sternum jutted into and deformed the anterior surface of the right ventricular free wall. These events were not affected by the interposition of 4 layers of paraffin gauze dressing but were hindered by the placement of a rigid barrier between the anterior portion of the heart and the inside of the thoracic wall.
\end{abstract}

Conclusion: The results show altered position of the heart in relation to the sternum during negative pressure wound therapy. This may explain 2 potentially hazardous events associated with negative pressure wound therapy, namely, risk for heart rupture and reduced cardiac output. Inserting a rigid barrier over the heart may be a protective measure that is clinically practicable.

Negative pressure wound therapy (NPWT) has remarkable effects on the healing of chronic and difficult wounds ${ }^{1-3}$ and has drastically reduced mortality in poststernotomy mediastinitis following cardiac surgery. ${ }^{4,5}$ The technique entails application of negative pressure to a sealed, airtight wound. The suction force created by the subatmospheric pressure enables the drainage of excessive fluid and debris, which leads to the removal of wound edema, reduction in bacterial count, and enhanced granulation tissue formation. ${ }^{6-9}$ Knowledge of the effects of NPWT in a sternotomy wound is limited. ${ }^{10}$ The organs in the mediastinum are hemodynamically crucial, and both vulnerable bypass grafts

From the Departments of Medicine, ${ }^{\mathrm{a}}$ Clinical Physiology, ${ }^{\mathrm{b}}$ and Cardiothoracic Surgery, ${ }^{\mathrm{c}}$ Lund University Hospital, Lund, Sweden; and Deutsches Herzzentrum Berlin, ${ }^{\mathrm{d}}$ Berlin, Germany.

This study was supported by the Åke Wiberg Foundation, the M. Bergvall Foundation, the Swedish Medical Association, the Royal Physiographic Society in Lund, the Swedish Medical Research Council, the Crafoord Foundation, the Swedish HeartLung Foundation, Lund University Faculty of Medicine, the Swedish Government Grant for Clinical Research and the Swedish Hypertension Society.

Received for publication Jan 3, 2008; revisions received Sept 9, 2008; accepted for publication Nov 16, 2008; available ahead of print July 6, 2009.

Address for reprints: Malin Malmsjö, MD, PhD, Vascular Research, Lund University, BMC A13, SE-221 84 Lund, Sweden (E-mail: malin.malmsjo@med.lu.se).

J Thorac Cardiovasc Surg 2009;138:712-7

$0022-5223 / \$ 36.00$

Copyright (c) 2009 by The American Association for Thoracic Surgery doi: $10.1016 /$ j.jtcvs. 2008.11 .068 and reduced cardiac function should be taken into consideration during NPWT of sternotomy wounds.

Recent publications have reported right ventricular rupture during NPWT in cardiac surgery. ${ }^{11,12}$ Also, reduced cardiac output during NPWT has been reported. ${ }^{13,14}$ Abu-Omar and colleagues ${ }^{11}$ speculated that heart rupture results from the overstretching of the right ventricle, which is adherent to the sternum and adjacent chest wall, during a sudden increase in intrathoracic pressure (eg, coughing or vomiting). No experimental study has yet been undertaken to morphologically explore the potential causes of heart rupture or decreased cardiac output during NPWT. The present study aimed to examine the effects of NPWT on the intrathoracic anatomy, in particular the heart, using real-time magnetic resonance imaging (MRI) in a porcine sternotomy wound model.

\section{MATERIALS AND METHODS Animals}

An uninfected porcine sternotomy wound model was used for the present study. Six domestic Landrace pigs of both genders, approximately 3 months of age, with a mean body weight of $50 \mathrm{~kg}$, were fasted overnight with free access to water. The study was approved by the Ethics Committee for Animal Research, Lund University, Sweden. The investigation complied with the "Guide for the Care and Use of Laboratory Animals" as recommended by the US National Institutes of Health and published by the National Academies Press (1996). 


\section{Abbreviations and Acronyms}

NPWT $=$ negative pressure wound therapy

MRI = magnetic resonance imaging

\begin{abstract}
Anesthesia and Surgical Procedure
Ketamine and xylazin were used for premedication. An infusion of propofol and fentanyl was given to maintain anesthesia, and atracurium besylate was given to achieve muscle paralysis. The pigs were surgically prepared for NPWT. A midline sternotomy was performed and the pericardium was opened. The left thoracic artery was harvested to mimic coronary artery bypass graft surgery. Thereafter, the sternotomy wound was prepared for NPWT. A polyurethane foam dressing was placed between the sternal edges and 2 noncollapsible drainage tubes were inserted into the foam. The open wound was then sealed with a transparent adhesive drape. The drainage tubes were connected to a purpose-built vacuum source (VAC pump unit, KCI, Copenhagen, Denmark), which was set to deliver a continuous negative pressure of $-75,-125$ or $-175 \mathrm{~mm} \mathrm{Hg}$. The NPWT experiments were initiated immediately after sternotomy. After the experiments were completed, the pigs were killed by an overdose of potassium. For details, see Wackenfors and colleagues, ${ }^{15}$ who used identical settings.
\end{abstract}

\section{Magnetic Resonance Imaging}

MRI was undertaken using a 1.5-T system (Philips Medical Systems, Best, The Netherlands) with a 5-element cardiac coil and the pig in the supine position. Imaging was undertaken during ventilator-controlled breathing using a real-time, steady-state free-precession sequence typically employing the following imaging parameters: repetition time/echo time 2.7/ 1.3 milliseconds, voxel size $1.37 \times 1.37 \times 10 \mathrm{~mm}$, SENSE reduction factor 2 and temporal resolution 100 milliseconds.

\section{Experimental Procedure}

MRI was performed from the onset of negative pressure and terminated when steady state was reached, which typically occurred within 2 minutes. MRI was performed in a midventricular transverse or a midsagittal plane during the application of NPWT at $-75,-125$, and $-175 \mathrm{~mm} \mathrm{Hg}$. To eliminate time effects, the sequence of applying the 3 different negative pressures $(-75,-125$, and $-175 \mathrm{~mm} \mathrm{Hg})$ was varied between the animals using a 3-by3 Latin square design. Imaging was performed before and after the insertion of 3 different devices: (1) Four layers of paraffin gauze dressing were placed over the anterior part of the heart (Jelonet, Smith \& Nephew, Hull, United Kingdom). (2) A $10 \times 20 \times 0.2-\mathrm{cm}$ perforated plastic rigid barrier was placed inside the thorax between the heart and the sternal edges. (3) A 1$\mathrm{cm}$-thick open porous structure material was placed underneath and around the heart with preserved communication to the intersternal foam to facilitate pressure transduction to the bottom of the wound. This piece of foam was cut from the manufactured black VAC foam (VAC black foam, KCI, Copenhagen, Denmark). The third device was constructed for the present study to examine the hypothesis that uneven pressure transduction in the thoracic cavity during NPWT (unpublished results) may contribute to the heart being displaced toward the anterior thoracic wall.

\section{RESULTS}

\section{Displacement of the Heart}

Prior to the application of NPWT, the heart was located centrally in the thorax and was clearly separated by air from the anterior thoracic wall (Figures 1 and 2). When negative pressure was applied, the following events could be clearly observed in all pigs studied. The air separating the heart from the thoracic wall was evacuated, whereby the heart was displaced toward the anterior thoracic wall. Furthermore, the movement of the anterior myocardium in the left and right ventricles was altered as the right ventricular free wall became fixed to and assumed the shape of the thoracic wall. Upon application of NPWT, the diastasis between the sternal edges decreased, and the polyurethane foam was compressed. The transverse (Figure 1) and midsagittal (not shown) imaging planes, from consecutive image acquisitions, showed the immediate proximity between the entire heart, encompassing the left and right ventricles from the base to the apex and the anterior thoracic wall following the application of negative pressure.

\section{Effects of Different Pressures}

No apparent differences in heart displacement could be observed with regards to the different magnitudes of negative pressure $(-75,-125$ or $-175 \mathrm{~mm} \mathrm{Hg})$.

\section{Potentially Hazardous Events}

In 2 pigs, the anterior portion of the right ventricular wall was displaced and bulged into the diastasis between the sternal edges (Figure 1). In 1 pig, the ribs on the left side were affected during surgery, at the time of harvesting the left internal thoracic artery, and did not oppose at the same level. This resulted in a sharp and uneven edge of the split sternum protruding into the thoracic cavity. Upon application of NPWT, the transverse imaging plane revealed how the heart was displaced toward the anterior thoracic wall, and the left hemisternum jutted into and deformed the anterior surface of the heart (Figure 1).

\section{Interface Dressings}

The effect of different interface dressings on the protection of the heart was examined during NPWT. Imaging was performed before and after the insertion of 3 different devices:

1. Four layers of paraffin gauze dressing were placed over the anterior portion of the heart. This slightly separated the heart from the anterior thoracic wall but did not prevent the deformation of the heart upon application of NPWT (Figure 2).

2. A $10 \times 20 \times 0.2-\mathrm{cm}$, perforated plastic rigid barrier was placed inside the thorax between the anterior surface of the heart and the thoracic wall. In the presence of this device, the heart still approached the anterior thoracic wall upon application of NPWT but was clearly separated from the sharp sternal edges. Also, the shape of the heart was not affected by NPWT (Figure 2).

3. An open porous structure material $(1 \mathrm{~cm}$ thick) was placed underneath the heart with preserved communication to the intersternal foam. This device was aimed to facilitate pressure transduction from the VAC foam between the sternal edges to the bottom of the wound. 




FIGURE 1. Potentially hazardous events: representative image frames in a midventricular transverse plane from real-time magnetic resonance imaging of negative pressure wound therapy (NPWT) of a pig sternotomy wound. Single images were selected from the time period before and upon achieving the target pressure of $-125 \mathrm{~mm} \mathrm{Hg}$. Images were acquired with no interface dressing or intrathoracic device. The images show how the heart is displaced toward the anterior thoracic wall while pressure is applied. This pattern was clearly visualized in all 6 pigs. The top panels (Example 1) show an example of the left hemisternum jutting into and deforming the anterior surface of the heart (arrow). This finding was particularly prominent in this pig. The bottom panels (Example 2) show an example of the anterior portion of the right ventricular wall displaced and bulging into the diastasis between the sternal edges (arrow). This pattern was clearly visualized in 2 of the pigs.

In the presence of this open porous structure material, the heart was not sucked as far up toward the anterior thoracic wall (Figure 3).

\section{DISCUSSION}

In the current study, real-time MRI revealed changes in the position of the right ventricular free wall in relation to the sternum, which occur during NPWT application, regardless of the level of negative pressure applied $(75,125$ or 175 $\mathrm{mm} \mathrm{Hg}$ ). The right ventricular free wall was displaced toward and fixed to the anterior thoracic wall. In some cases, NPWT caused the heart to bulge into the space between the sternal edges, and the sharp edges of the sternum jutted into and deformed the anterior surface of the heart. The present study provides a plausible mechanism for 2 potentially hazardous events associated with NPWT, namely, heart rupture and reduced cardiac output. Furthermore, the insertion of a rigid barrier, but not paraffin gauze, between the heart and the thoracic wall appears to prevent the deformation of the heart.

\section{Heart Rupture}

The present study shows that the heart is displaced during the application of negative pressure. This may cause a lacer- ation of the free wall of the right ventricle or of a bypass graft. Patients with mediastinitis and vulnerable tissue may be especially susceptible. Therefore, it might be necessary to combine NPWT with a device that protects the heart. Currently, this is performed by placing multiple layers of paraffin gauze over the anterior portion of the heart. However, the present results show that this procedure does not hinder the heart from being displaced and deformed by the sternal edges.

\section{Cardiac Pumping}

NPWT has been shown to result in an immediate decrease in cardiac output, stroke volume, and left ventricular end-diastolic volume. ${ }^{13,14}$ The present observations may provide insight into these previous findings. Cardiac pumping may be affected when the heart is displaced toward the anterior thoracic wall and the movement of the myocardium is altered. The results from the present study indicate that NPWT alters the intrathoracic anatomy, which may mechanically impair the contraction and/or relaxation of the ventricles. The negative effect of NPWT on cardiac pumping does not seem to depend on the level of negative pressure applied $(75,125$ or $175 \mathrm{~mm} \mathrm{Hg}) .{ }^{14}$ Also, the present results indicate that the change in intrathoracic anatomy is similar during the application of these 3 different levels of negative pressure. 


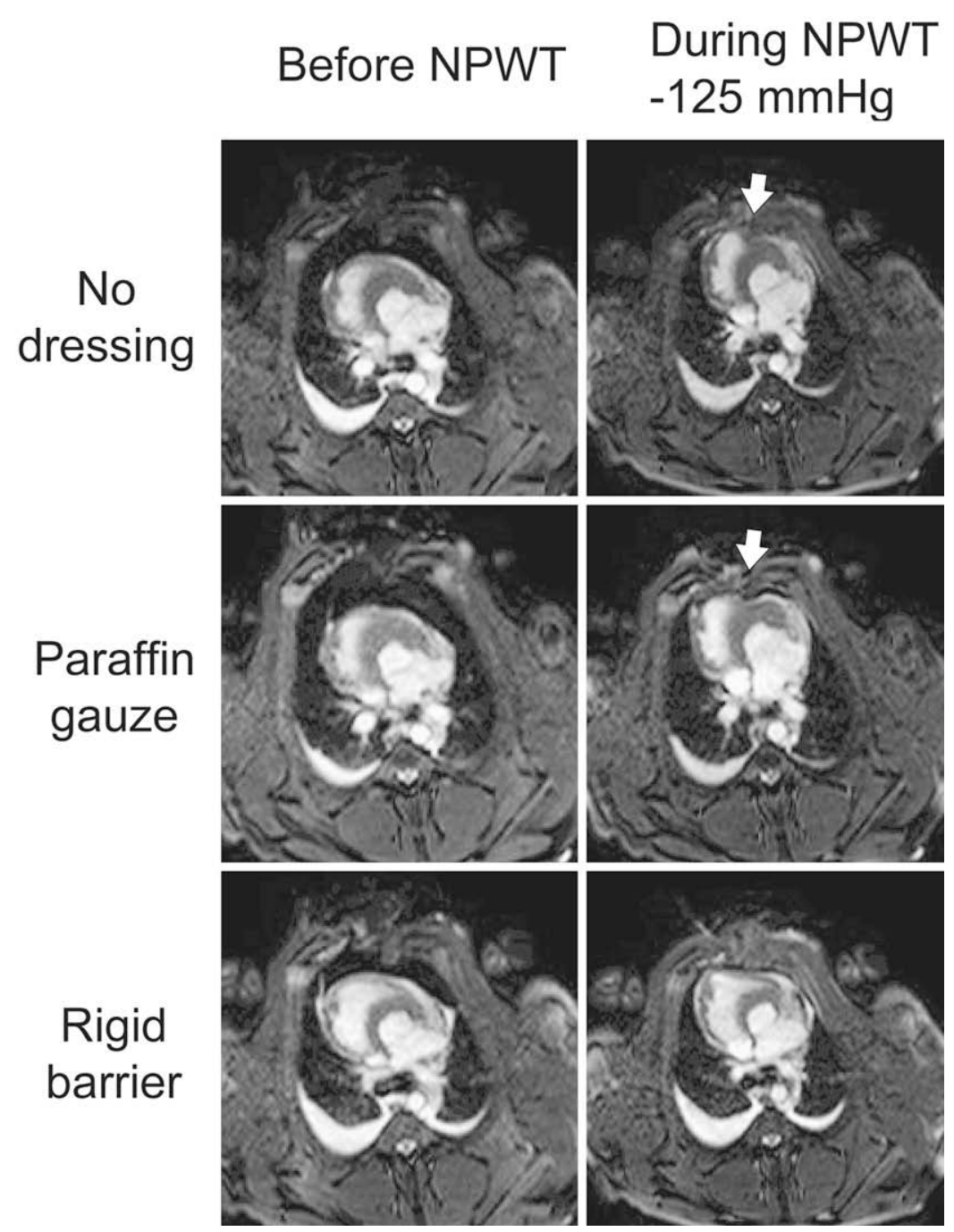

FIGURE 2. Effects of paraffin gauze or rigid barrier: representative image frames in a midventricular transverse plane from real-time magnetic resonance imaging of negative pressure wound therapy $(N P W T)$ of a pig sternotomy wound. Single images were selected from the time period before and upon achieving the target pressure of $-125 \mathrm{~mm} \mathrm{Hg}$. Images were acquired with no dressing, with paraffin gauze interface dressing, and with a rigid barrier between the heart and the anterior thoracic wall, respectively. Note how the mechanical deformation of the heart upon NPWT application is prevented by a rigid barrier but not by a paraffin gauze interface dressing.

\section{Pressure Transduction to the Bottom of the Wound}

We observed that the pressure, during NPWT, is transduced only to the anterior surface of the heart, but not to places that are not in direct contact with the VAC foam, further down in the thoracic cavity (eg, in the pericardium under the heart, in the left pleura, and in the esophagus; unpublished results). In the present study, a 1-cm-thick open porous structure material was placed underneath the heart, with preserved communication to the intersternal foam, to facilitate pressure transduction from the intersternal foam to the bottom of the wound. In the presence of this open porous structure material, the heart was not sucked as far up toward the anterior thoracic wall and did not end up in the intersternal space at the place for the sharp sternal edges. We assume that the pressure difference between the anterior and poste- rior portions of the heart causes the right ventricle to be displaced toward the posterior parts of the sternum.

Obviously, this procedure of inserting foam underneath the heart is inapplicable in clinical practice because of distinct adhesions around the heart in a patient with mediastinitis. The device was constructed for the present study to examine the hypothesis that uneven pressure transduction in the thoracic cavity during NPWT may contribute to the heart moving toward the anterior thoracic wall.

\section{Rigid Barrier}

MRI shows that interposition of a rigid barrier inside the thorax, between the anterior surface of the heart and the thoracic wall, hinders the heart from being displaced or deformed by the sternal edges. We speculate that such a device may 


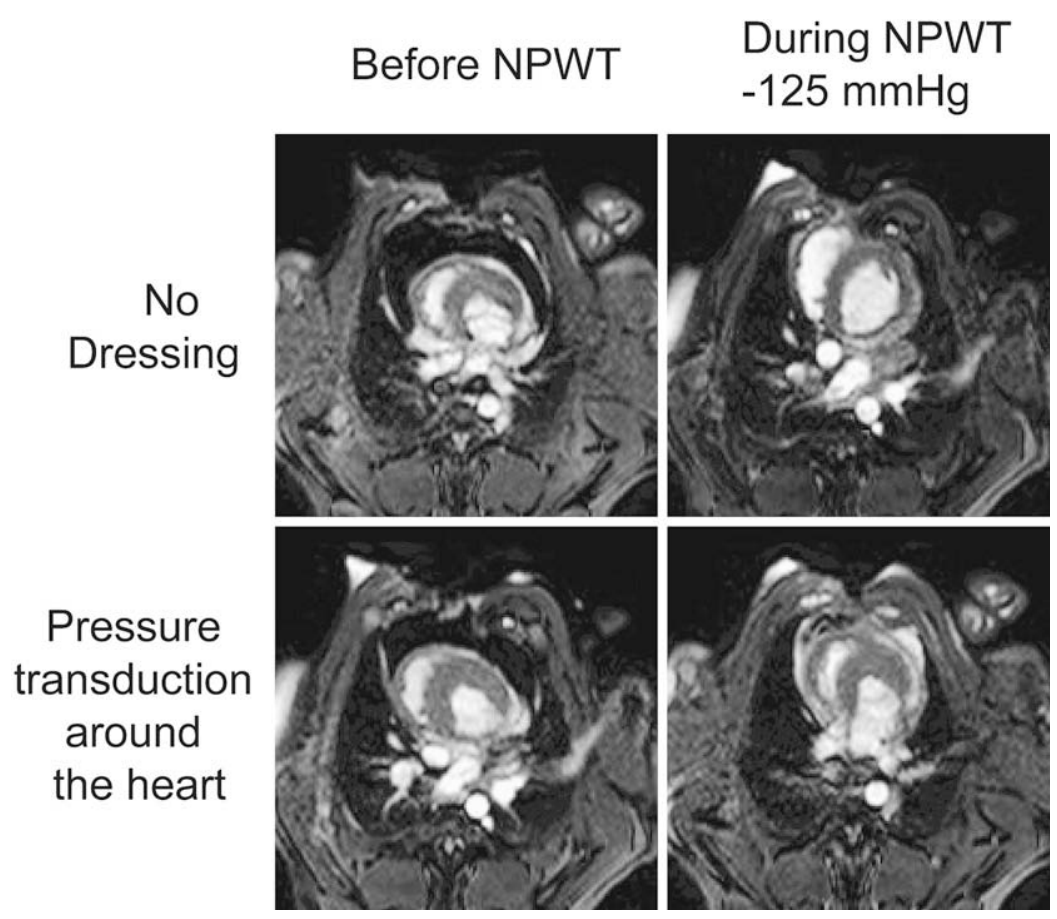

FIGURE 3. Pressure transduction to the bottom of the wound: representative image frames in a midventricular transverse plane from real-time magnetic resonance imaging of negative pressure wound therapy $(N P W T)$ of a pig sternotomy wound. Single images were selected from the time period before and upon achieving the target pressure of $-125 \mathrm{~mm} \mathrm{Hg}$. Images were acquired with no dressing and with a 1-cm-thick, open porous structure material placed underneath and around the heart with preserved communication to the intersternal foam to facilitate pressure transduction to the bottom of the wound. Note that the heart is not sucked as far up when there is preserved communication to the bottom of the wound.

prevent heart rupture and cardiac output reduction during NPWT. Indeed, covering the heart with a wound interface dressing has been shown previously to lessen the negative hemodynamic effects of NPWT. ${ }^{14}$ The procedure of inserting a rigid barrier to protect the heart may be clinically practicable. However, adhesions in patients with mediastinitis presumably require caution during insertion. Furthermore, vulnerable bypass grafts need to be taken into consideration.

\section{Limitations of the Study}

We show that the heart is displaced toward the sharp sternal edges during NPWT. This is a plausible mechanism for cardiac rupture in the setting of NPWT technique. However, the pig model has limitations. Patients are subject to negative pressure for days, although the pigs were only subject to negative pressure for brief moments, and there was no confirmation of heart injury in the present study. Another limitation is that this study was conducted on $50-\mathrm{kg}$ pigs and NPWT in the clinic is performed on $70-$ to $100-\mathrm{kg}$ patients. The anatomy of the pig chest is also different from that of humans. The porcine chest has a shorter lateral diameter and a wider anteroposterior diameter relative to humans, which may play a role for the degree of possible anterior displacement of the heart. Also, the amount of relative subcutaneous tissues in humans is quite different from that of a $50-\mathrm{kg}$ pig. Furthermore, the present study was performed in a noninfected pig model of sternotomy, which is different from the clinical setting. In the majority of patients, sternal dehiscence, sternal infection, or mediastinitis is a delayed finding, 8 to 32 days postoperatively by one report. ${ }^{8}$ At this time, adhesions have already formed and the heart's position is somewhat fixed in the mediastinum, at least to some extent depending on the severity of infection. Although the present study demonstrated anterior cardiac displacement in pigs after immediate NPWT, it remains to be observed whether such a displacement occurs in humans, 1 week or more after heart surgery. An alternative would be to perform imaging 5 to 9 days after NPWT in pigs. The situation in the thoracic cavity may be different after this time, with a formation of adhesions. However, such survival experiments on pigs undergoing sternotomy wound NPWT would need special ethical considerations. It should also be kept in mind that MRI was performed during ventilator-controlled breathing. Not all human patients with NPWT require positive pressure ventilation. Positive pressure changes the transthoracic pressure. The combination of positive pressure in the pleural spaces and negative pressure of $125 \mathrm{~mm} \mathrm{Hg}$ at the chest may contribute to anterior cardiac displacement that may not be present or as significant in the nonventilated pressure that inspires with negative pleural pressure.

In conclusion, in the present study, real-time MRI during the application of NPWT revealed that the heart was 
displaced toward the anterior thoracic wall, and, in some cases, the right ventricular free wall was observed to bulge into the space between the sternal edges that jutted into the surface of the heart. This is a plausible mechanism that may explain heart rupture and decreased cardiac output during NPWT. Four layers of paraffin gauze dressing did not prevent the deformation of the heart. Inserting a rigid barrier over the anterior portion of the right ventricle clearly separated the heart from the sharp sternal edges and hindered deformation. This procedure may be clinically practicable and might provide a solution for problems associated with NPWT in cardiac surgery.

We thank Thomas Krabatsch, MD, PhD, and Christof Stamm, $\mathrm{MD}, \mathrm{PhD}$, at the Deutsches Herzzentrum Berlin, Berlin, Germany, for their valuable comments.

\section{References}

1. Argenta LC, Morykwas MJ, Marks MW, DeFranzo AJ, Molnar JA, David LR. Vacuum-assisted closure: state of clinic art. Plast Reconstr Surg. 2006;117(7 Suppl):127S-42S.

2. Banwell P, Teot L. Topical negative pressure (TNP): the evolution of a novel wound therapy. J Tissue Viability. 2006;16:16-24.

3. Obdeijn MC, de Lange MY, Lichtendahl DH, de Boer WJ. Vacuum-assisted closure in the treatment of poststernotomy mediastinitis. Ann Thorac Surg. 1999;68: 2358-60.

4. Crabtree TD, Codd JE, Fraser VJ, Bailey MS, Olsen MA, Damiano RJ Jr. Multivariate analysis of risk factors for deep and superficial sternal infection after coronary artery bypass grafting at a tertiary care medical center. Semin Thorac Cardiovasc Surg. 2004; 16:53-61.
5. Lu JC, Grayson AD, Jha P, Srinivasan AK, Fabri BM. Risk factors for sternal wound infection and mid-term survival following coronary artery bypass surgery. Eur J Cardiothorac Surg. 2003;23:943-9.

6. Argenta LC, Morykwas MJ. Vacuum-assisted closure: a new method for wound control and treatment: clinical experience. Ann Plast Surg. 1997;38:563-76; discussion 577.

7. Clare MP, Fitzgibbons TC, McMullen ST, Stice RC, Hayes DF, Henkel L. Experience with the vacuum assisted closure negative pressure technique in the treatment of non-healing diabetic and dysvascular wounds. Foot Ankle Int. 2002;23: 896-901.

8. Domkowski PW, Smith ML, Gonyon DL Jr, Drye C, Wooten MK, Levin LS, Wolfe WG. Evaluation of vacuum-assisted closure in the treatment of poststernotomy mediastinitis. J Thorac Cardiovasc Surg. 2003;126:386-90.

9. Morykwas MJ, Argenta LC, Shelton-Brown EI, McGuirt W. Vacuum-assisted closure: a new method for wound control and treatment: animal studies and basic foundation. Ann Plast Surg. 1997;38:553-62.

10. Malmsjö M, Ingemansson R, Sjögren J. Mechanisms governing the effects of vacuum-assisted closure in cardiac surgery. Plast Reconstr Surg. 2007;120:1266-75.

11. Abu-Omar Y, Naik MJ, Catarino PA, Ratnatunga C. Right ventricular rupture during use of high-pressure suction drainage in the management of poststernotomy mediastinitis. Ann Thorac Surg. 2003;76:974; author reply 974-5.

12. Sartipy U, Lockowandt U, Gabel J, Jideus L, Dellgren G. Cardiac rupture during vacuum-assisted closure therapy. Ann Thorac Surg. 2006;82:1110-1.

13. Conquest AM, Garofalo JH, Maziarz DM, Mendelson KG, Su Sun Y, Wooden WA, et al. Hemodynamic effects of the vacuum-assisted closure device on open mediastinal wounds. J Surg Res. 2003;115:209-13.

14. Petzina R, Ugander M, Gustafsson L, Engblom H, Sjogren J, Hetzer R, et al. Hemodynamic effects of vacuum-assisted closure therapy in cardiac surgery: assessment using magnetic resonance imaging. J Thorac Cardiovasc Surg. 2007;133: 1154-62.

15. Wackenfors A, Gustafsson R, Sjogren J, Algotsson L, Ingemansson R, Malmsjo M. Blood flow responses in the peristernal thoracic wall during vacuum-assisted closure therapy. Ann Thorac Surg. 2005;79:1724-30; discussion 1730-1. 